\title{
Prevention and Treatment of Viral Infections by Natural Therapies
}

\author{
Keywords: Viral Infection; Prevention; Alternative Therapy; Natural \\ Medication; Medicinal Herbs; Virus Eradication.
}

\section{Editorial}

Many viral infections are still causing tremendous threat to human being such as HIV, coronavirus, SARS, avian influenza, swine flu, Dengue virus, Ebola virus, etc. There are no efficient conventional medications or vaccines for most of these viruses. Consequently alternative natural medications are an urgent requirement to fill the gap of unavailability of conventional therapies or vaccines. Complementary and alternative medicine has been used for centuries in many societies to treat various illnesses, including viral infections. Herbal, dietary, complementary, and natural therapies have been used widely for prevention and treatment of viral infections. Some of these medications are introduced in the present article. Respiratory viruses are a major cause of influenza-like illness (ILI) symptoms in children and adults, leading to substantial morbidity and mortality each year. Several herbal extracts were proved effective for prevention and treatment of respiratory viral infections that based on scientific ground. Among these herbs are licorice roots, North American ginseng, berries, Echinacea, pomegranate, and guava tea. The active ingredients of these plants against flu and cold viruses are neuraminidase inhibitors, glycyrrhizin, polyphenol, baicalin, etc. The mechanism of actions that plants extracts could fight influenza through: neuraminidase inhibition, preventing virus budding, assistance of viral bounding to natural antibodies, stimulation of IFN-gamma production by $T$ cells, inhibition viral hemagglutination activity, inhibition viral binding to and penetration into host cells, enhancement production of antiinfluenza virus immunoglobulin, synthesis inhibition of both viral RNA and protein, replication suppression of influenza virus, secretion induction of type I IFN and pro-inflammatory cytokines with subsequent stimulation of the antiviral activity, and exertion virion structural damage [1]. Respiratory syncytial virus (RSV) is a common cause bronchiolitis in children that followed by inflammation and asthma-like symptoms. A preventive therapy for this virus has not been developed yet. Chang et al. investigated the anti-inflammatory function of fungal immunomodulatory protein on RSV. FIP-fve is an immunomodulatory protein isolated from Flammulina velutipes. The results of the study suggested that FIP-fve decreases RSV replication, RSV-induced inflammation

\section{Haider Abdul-Lateef Mousa \\ MB ChB, MSc, Lecturer, College of Medicine, University of Basrah, Iraq}

\author{
Corresponding Author: \\ Haider Abdul-Lateef Mousa \\ haideramousa@hotmail.com \\ PO Box 601, Post Code 42001, Ashar, \\ Basrah, Iraq
}

Tel: 009647808595467

and respiratory pathogenesis. The investigators concluded that a natural compound extracted from F.velutipes (FIP-fve) may be a safe agent for viral prevention and even therapy [2]. It is now recognized that an overwhelming inflammatory response is the cause of human deaths from infection with the avian H5N1 influenza [3]. Earthing or grounding (connecting the human body to the earth) has demonstrated anti-inflammatory effects in human body and improvements in the immune response that both effects might assist in influenza treatment [4]. Diarrhoea associated with rotavirus is one of the major gastrointestinal problems faced by human infants. The protective effectiveness of probiotic feeding against naturally acquired diarrhoea is under investigation. Evidence suggests that specific probiotics may be antagonistic to enteric viruses and enhance immunity, and thus, provide a means of preventing or treating diarrheal diseases. In a clinical trial including twenty-nine pediatric patients who presented with acute rotaviral gastroenteritis. Six probiotic strains (Bifidobacterium longum, B. lactis, Lactobacillus acidophilus, L. rhamnosus, L. plantarum, and Pediococcus pentosaceus) were given to the patients twice daily for 1 week. The probiotics were found significantly shortened the duration of diarrhoea in these patients as compared with a placebo. The study revealed that the probiotics may be useful for the treatment of acute rotaviral gastroenteritis or as an alternative therapy without adverse effects [5]. Dengue fever is caused by the mosquitoborne dengue virus (serotypes 1-4) which is the most common arboviral infection of humans in tropical and subtropical regions of the world. Tong et al investigated the effect of herb known as Gastrodia elata on the multiplication cycle of dengue virus serotype 2 (DV2). The investigators concluded that the active ingredient of the herb (WSS45) exerted potent inhibitory effect on DV2 through interfering with the interaction between viruses and targeted cells [6]. Kiat et al. investigated the effect of Chinese 
ginger or finger root on dengue 2 virus. Their study showed good competitive inhibitory activities towards dengue 2 virus NS3 protease [7]. Medical ozone revealed bactericidal, fungicidal, and virucidal activity. Some studies proved that ozone infused into donated blood samples can kill viruses into a higher extent of cases. Zaky et al. studied the effect of ozone therapy in patients with chronic hepatitis $C$. The investigators concluded that ozone therapy significantly improved the clinical symptoms associated with chronic hepatitis $C$ and was associated with normalized ALT and AST levels among a significant number of patients. Ozone therapy also demonstrated disappearance of HCV RNA from the serum (negative PCR for HCV RNA) in $25 \%-45 \%$ of patients with chronic hepatitis $\mathrm{C}$ [8]. Traditional Korean herbs (known as $\mathrm{KYH}$ 1) have been recognised that exhibit potency against hepatitis $B$ virus and hepatitis $C$ virus infections. The antiviral activity of an aqueous extract of herbal formulation $\mathrm{KYH}-1$ was tested in tissue culture systems for HBV and HCV. The researchers showed that $\mathrm{KYH}-1$ exhibited potent antiviral activity against hepatitis viruses through inhibition HBV polymerase and suppressing viral replication [9]. 


\section{References}

1 Mousa HA (2015) Herbal Therapies for Prevention and Treatment of Influenza and Influenza-Like Illness. J Infect Dis Ther 3: 215.

2 Chang YC, Chow YH, Sun HL, Liu YF, Lee YT(2014) Alleviation of respiratory syncytial virus replication and inflammation by fungal immunomodulatory protein FIP-fve from Flammulina velutipes. Antiviral Res 110: 124-131.

3 Alleva LM, Cai C, Clark IA (2010) Using complementary and alternative medicines to target the host response during severe influenza. Evid Based Complement Alternat Med 7: 501-510.

4 Mousa HA, Health impacts of alkaline diet and water, reduction of digestive-tract bacterial load, and earthing. Alternative Therapies in Health and Medicine in press.

5 Lee do K, Park JE, Kim MJ, Seo JG, Lee JH, et al. (2015) Probiotic bacteria, $\mathrm{B}$. longum and $\mathrm{L}$. acidophilus inhibit infection by rotavirus in vitro and decrease the duration of diarrhea in pediatric patients. Clin Res Hepatol Gastroenterol 39: 237-244.

6 Tong XK, Qiu H, Zhang X, Shi LP, Wang GF, et al. (2010) WSS45, A sulfated alpha-D-glucan, strongly interferes with Dengue 2 virus infection in vitro. Acta Pharmacol Sin 31: 585-592.

7 Kiat TS, Pippen R, Yusof R, Ibrahim H, Khalid N, et al. (2006) Inhibitory activity of cyclohexenyl chalcone derivatives and flavonoids of fingerroot, Boesenbergia rotunda (L.), towards dengue-2 virus NS3 protease. Bioorg Med Chem Lett. 16: 3337-3340.

8 Zaky S, Kamel SE, Hassan MS, Sallam NA, Shahata MA (2011) Preliminary results of ozone therapy as a possible treatment for patients with chronic hepatitis C. J Altern Complement Med 17: 259263.

9 Jacob JR, Korba BE, You JE, Tennant BC, Kim YH (2004) Korean medicinal plant extracts exhibit antiviral potency against viral hepatitis. J Altern Complement Med 10: 1019-1026. 